\title{
Threshold Current Density for Perpendicular Magnetization Switching Through Spin-Orbit Torque
}

\author{
Daoqian $\mathrm{Zhu}^{1,2}$ and Weisheng Zhao ${ }^{1,2, *}$ \\ ${ }^{1}$ Fert Beijing Research Institute, BDBC, Beihang University, Beijing 100191, China \\ ${ }^{2}$ School of Microelectronics, Beihang University, Beijing, 100191, China
}

(Received 15 December 2019; revised manuscript received 30 March 2020; accepted 3 April 2020; published 30 April 2020)

\begin{abstract}
We theoretically investigate the threshold current density for spin-orbit-torque- (SOT) induced perpendicular magnetization switching. According to the relative sign of fieldlike torque (FLT) and dampinglike torque (DLT), we derive the corresponding analytical formulas of the threshold current density $J_{\text {th }}$ required to switch magnetization from equilibrium states to nearly in-plane states. These formulas, which agree well with numerical results among a wide range of material parameters, indicate that SOT current density can be significantly reduced in the presence of a large FLT. The conditions are then explored to achieve complete magnetization reversal after removing the SOT currents. When the ratio of FLT to DLT $\eta$ is negative in our sign convention, we find that SOT pulses with long enough fall time, for example, $0.2 \mathrm{~ns}$ in our simulations, can guarantee stable switching. In contrast, a small in-plane magnetic field or a large damping is necessary when $\eta>0$. Based on the conditions, the measured threshold current densities in several reported experiments, which deviate from previous models, can be well reproduced by our derived $J_{\text {th }}$. We further study the possible incubation delay induced by SOT, clarifying the recent contradiction on incubation time during SOT switching. Our work sheds insights on the SOT material optimization and the comprehension of magnetization switching dynamics induced by SOT.
\end{abstract}

DOI: 10.1103/PhysRevApplied.13.044078

\section{INTRODUCTION}

Manipulation of magnetization states is the central topic of magnetoresistive random-access memory (MRAM) that emerges as a promising solution to the power dissipation issue in the post Moore era [1-5]. Broad attention has been focused on using spin-polarized currents, rather than magnetic fields, to deliver spin angular momentum to ferromagnets and induce bidirectional magnetization switching because of its potential to realize ultradense and energy-efficient memories [3]. One approach is to apply a vertical current into a magnetic tunnel junction (MTJ), the MRAM cell. This current is polarized by the pinned ferromagnetic layer, thus exerting spin-transfer torque (STT) to switch the magnetization of the free layer [6-8]. Over the years, STT MRAM has been widely investigated and proven to achieve reliable data writing within $5 \mathrm{~ns}$ at power consumption of about $1 \mathrm{pJ}$ [9], which is attractive in embedded applications. Nevertheless, there are still several

\footnotetext{
*weisheng.zhao@buaa.edu.cn

Published by the American Physical Society under the terms of the Creative Commons Attribution 4.0 International license. Further distribution of this work must maintain attribution to the author(s) and the published article's title, journal citation, and DOI.
}

challenges to be tackled for STT MRAM, such as the barrier breakdown due to the high tunneling currents through the $\mathrm{MgO}$ barrier [10] and the long incubation delay caused by the collinear direction of free-layer magnetization and spin polarization [11].

As an alternative, spin-orbit torque (SOT) has been exploited to address the bottlenecks of the STT scheme [12-17]. By injecting a charge current into materials with strong spin-orbit coupling, such as heavy-metal (HM) [12-14,16,17] and topological insulators [15], a spin (-polarized) current generates and exerts SOT to the adjacent ferromagnetic (FM) layer to induce magnetization switching. The endurance of MTJs can be improved as there is no large tunneling current passing through the barrier. In addition, no incubation delay was observed in $\mathrm{Pt} / \mathrm{Co} / \mathrm{AlO} x[16,17]$ and reliable subnanosecond SOT switching has been realized [16-20], which is of considerable interest in ultrafast spintronic memories.

Despite the success in experiments, on the one hand, the physical mechanism of SOT still remains to be clarified [21]. It is generally accepted that SOT is composed of two components, namely, the dampinglike torque (DLT) and the fieldlike torque (FLT) [22]. As both torques can be generated by bulk or interfacial effects [21-25], it is hard to distinguish which effect is dominant, thus efforts have long been devoted to interpret it. On the other hand, to further 
optimize switching performance, it is necessary to investigate the threshold current density $J_{\text {th }}$ for SOT-induced perpendicular magnetization switching. We focus on ferromagnets with perpendicular magnetic anisotropy (PMA) here because of their scalability down to $20 \mathrm{~nm}$ diameter [7]. In 2013, Lee et al. derived an analytic formula with only DLT considered [26]:

$$
J_{\mathrm{th}}=\frac{e t_{F} \mu_{0} M_{s}}{\hbar \theta_{\mathrm{SH}}}\left(H_{K, \mathrm{eff}}-\sqrt{2} H_{x}\right)
$$

where $H_{K \text {,eff }}$ is the effective anisotropy field, $M_{s}$ is the saturation magnetization, $t_{F}$ is the thickness of the free layer, $\theta_{\mathrm{SH}}$ is the spin Hall angle describing the charge-to-spin conversion efficiency, $e$ is the elementary charge, and $\hbar$ is the reduced Planck constant. $H_{x}$ is the applied in-plane magnetic field to break the symmetry of SOT and several schemes have been proposed to eliminate it $[4,5,27-31]$. Equation (1) has been widely adopted to analyze experiments but it is also necessary to include the effect of FLT since it cannot be neglected in the widely studied HMFM-oxide trilayer structure [17-19,27]. In 2015, Taniguchi et al. obtained the SOT threshold current density when both DLT and FLT are present and the ratio of FLT to DLT $\eta$ is positive in our sign convention [32] [see Eq. (3)]:

$$
J_{\mathrm{th}}=\sqrt{\frac{8 \alpha}{\eta(2+\alpha \eta)}} \frac{e t_{F} \mu_{0} M_{s} H_{K, \mathrm{eff}}}{\hbar \theta_{\mathrm{SH}}} \sqrt{1-\frac{1}{2}\left(\frac{H_{x}}{H_{K, \mathrm{eff}}}\right)^{2}},
$$

where $\alpha$ is the damping constant. This formula is in good agreement with numerical results in the low damping and strong FLT region, but simulations show that Eq. (2) will become inaccurate when $\eta$ is small and $\alpha$ is strong, like $\eta=0.4$ and $\alpha=0.08$. To date, an analytical formula of $J_{\text {th }}$ that is accurate among a wide range of material parameters is still lacking and the $\eta<0$ case has not been studied yet. It is also worth noting that Eqs. (1) and (2) are threshold current densities derived from the instability condition, i.e., driving magnetization from equilibrium states to nearly inplane states. Magnetization switching back after removing SOT currents remains as an issue because the magnetization relaxation dynamics become complex in the presence of the applied in-plane magnetic field [26,33]. Taniguchi has given the criteria to realize stable switching when only DLT is included [33], but the case where the FLT exists remains unexplored.

In this paper, we firstly investigate the analytical expressions of the threshold current density $J_{\text {th }}$ to switch magnetization from $+z$ direction to nearly in-plane states with $-z$ component. According to the relative sign of DLT and FLT, which determines whether SOT competes with damping [32], two different methods are adopted to derive the formulas. Numerical simulations demonstrate the high accuracy of the formulas among a wide range of material parameters. We then calculate the phase diagram of $z$ component of magnetization after removing SOT currents to explore the conditions for stable full switching. The results suggest that SOT pulses with long enough fall time can facilitate stable switching when $\eta<0$ while a small in-plane magnetic field or a large damping is required if $\eta>0$. With the conditions satisfied, the derived formulas are applied to interpret the measured threshold current densities in several experiments. We further discuss the possible incubation delay during SOT switching, which is verified by micromagnetic simulations and can be used to interpret the phenomena observed in recent experiments $[16,17,34]$.

\section{METHOD}

\section{A. Macrospin and micromagnetic simulations}

Figure 1 shows the schematic of a typical three-terminal SOT device, where an MTJ is on the top of a bottom layer (SOT channel). An in-plane SOT current $J_{\text {SOT }}$ is injected into the bottom layer to switch the adjacent FM layer with PMA. The in-plane magnetic field $H_{x}$ is applied to achieve deterministic SOT switching. Within singledomain approximation, the magnetization dynamics can be depicted by the following Landau-Lifshitz-Gilbert (LLG) equation [35]:

$$
\begin{aligned}
\frac{\partial \mathbf{m}}{\partial t}= & -\gamma \mu_{0}\left(\mathbf{m} \times \mathbf{H}_{\mathbf{K}, \text { eff }}\right)-\gamma \mu_{0}\left(\mathbf{m} \times \mathbf{H}_{\mathbf{x}}\right) \\
& +\alpha\left(\mathbf{m} \times \frac{\partial \mathbf{m}}{\partial t}\right)+\gamma \mu_{0} H_{\mathrm{SOT}}^{\mathrm{DL}}(\mathbf{m} \times \boldsymbol{\sigma} \times \mathbf{m}) \\
& +\gamma \mu_{0} H_{\mathrm{SOT}}^{\mathrm{FL}}(\mathbf{m} \times \boldsymbol{\sigma}),
\end{aligned}
$$

where the first two terms at the right side of Eq. (3) are the torques originating from the effective anisotropy field and the in-plane magnetic field, respectively. The third term denotes the damping torque. The rest two terms represent the DLT and FLT of SOT, respectively. $\mathbf{m}=\mathbf{M} / M_{s}$ is the reduced magnetization, $\gamma$ is the gyromagnetic ratio, $\mathbf{H}_{\mathbf{K} \text {,eff }}=H_{K, \text { eff }} m_{z} \hat{\mathbf{z}}$ is the effective anisotropy field, including the contributions from the anisotropy field and the demagnetization field, $\mathbf{H}_{\mathbf{x}}=H_{x} \hat{\mathbf{x}}$ is the external in-plane magnetic field and $\sigma$ is the spin-polarization direction of SOT. $H_{\mathrm{SOT}}^{\mathrm{DL}}=\left(J_{\mathrm{SOT}} \theta_{\mathrm{SH}} \hbar\right) /\left(2 e t_{F} \mu_{0} M_{s}\right)$ and $H_{\mathrm{SOT}}^{\mathrm{FL}}=\eta H_{\mathrm{SOT}}^{\mathrm{DL}}$ are the corresponding field strength for the DLT and FLT, respectively. The ratio of FLT to DLT $\eta$ can vary a lot even in the same material system, affected by HM-layer thickness [36], oxidation [37], and annealing [38], etc. In the micromagnetic simulations [39], the magnetization dynamics at each site follow Eq. (3), with Heisenberg exchange field and Dzyaloshinskii-Moriya interaction (DMI) field included. Simulated regions are discretized into $2 \times 2 \times 1 \mathrm{~nm}^{3}$ grid. Demagnetization field is turned 


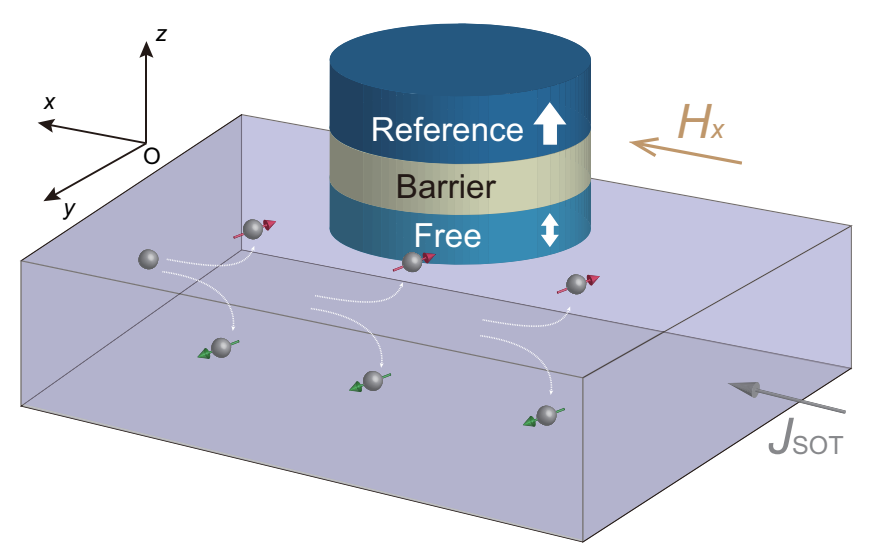

FIG. 1. Schematic of a three-terminal SOT device.

off in the micromagnetic simulations to ensure the same anisotropy field as in the macrospin simulations since the demagnetizing factor is hard to accurately estimate in the simulated region. In our simulations, SOT pulses of $300 \mathrm{~ns}$ are applied to ensure the quasistable states. The SOT current density is considered exceeding the threshold when $m_{z}<0$ at $t=300 \mathrm{~ns}$. All the simulations are performed at $T=0 \mathrm{~K}$ as the effect of thermal agitation is not the focus of this work [40].

Unless specified, the material parameters adopted in this paper are as follows: saturation magnetization $M_{s}=1200 \mathrm{KA} \mathrm{m}{ }^{-1}$, effective anisotropy field $\mu_{0} H_{K, \text { eff }}=0.4 \mathrm{~T}$, free-layer thickness $t_{F}=1 \mathrm{~nm}$, which contributes to a thermal stability of 41 at room temperature for MTJs with a radius of $15 \mathrm{~nm}$. Gyromagnetic ratio $\gamma=1.76 \times 10^{11} \mathrm{~T}^{-1} \mathrm{~s}^{-1}$, spin Hall angle $\theta_{\mathrm{SH}}=0.3$, exchange stiffness $A=15 \mathrm{pJ} \mathrm{m}^{-1}$, damping constant $\alpha$ varies from 0.004 to 0.128 and FLT to DLT ratio $\eta$ varies from -8 to 3 . The spin-polarization direction of SOT is $\sigma=(0,-1,0)$. The initial magnetization $\mathbf{m}_{0}$ is set as $(0,0,1)$.

\section{B. Derivation of formulas}

Two methods are adopted to derive the formulas based on the sign of $\eta$, which determines the competition between SOT and damping. We first consider the $\eta>0$ case, following the method of Ref. [32]. Because SOT competes with damping directly under this circumstance, magnetization is found to precess around an effective field initially. Once the applied current exceeds the threshold value, the precession amplitude gradually increases, thus destabilizing the magnetization from $+z$ direction and vice versa. Therefore, the threshold current density can be determined from this condition. The change rate of magnetization component along the effective-field direction is slow at the beginning, hence this component can be regarded as a constant. As a result, the LLG equation can be linearized to a second-order ordinary differential equation in a new coordinate with the $z$ axis along the effective-field direction and then be solved analytically.

Based on the above discussion, it is critical to determine the effective field $\mathbf{H}_{\text {eff }}$, which directly influences the calculation accuracy. According to the form of different torques in Eq. (3), it may be intuitive to choose $\mathbf{H}_{\mathbf{K} \text {,eff }}+$ $\mathbf{H}_{\mathbf{x}}-H_{\mathrm{SOT}}^{\mathrm{FL}} \boldsymbol{\sigma}$ as $\mathbf{H}_{\text {eff }}$, as adopted in Ref. [32]. However, we argue that the DLT can also contribute a component, $-H_{\mathrm{SOT}}^{\mathrm{DL}} \boldsymbol{\sigma} \times\langle\mathbf{m}\rangle$, where $\langle\mathbf{m}\rangle$ denotes the time average of magnetization $\mathbf{m}$ during the initial precessions. Because $\langle\mathbf{m}\rangle$ has at least a $+z$ component, the DLT in Eq. (3) will definitely give rise to a field component along $+x$ direction. This is in marked contrast to the STT switching whose spin polarization $\mathbf{m}_{p}$ is collinear with magnetization. Consequently, $\mathbf{m}_{p} \times \mathbf{m}$ is zero, resulting in no contributions to the effective field. Figure 2(a) shows the tridimensional precession trajectory of $\mathbf{m}$ with $J_{\mathrm{SOT}}=68 \mathrm{MA} \mathrm{cm}^{-2}\left(J_{\text {th }}\right.$ is $\left.69 \mathrm{MA} \mathrm{cm}^{-2}\right), \alpha=0.1$, and $\eta=3$. The trajectory projecting on the $x-y$ plane is presented in Fig. 2(c). The in-plane field $H_{x}$ is removed to avoid interference. Since $J_{\mathrm{SOT}}$ is lower than the threshold, magnetization $\mathbf{m}$ precesses around an effective field firstly and eventually damps into $(0.128,0.429,0.894)$ (the SOT current is still applied), as indicated by the red star in Fig. 2(c). Obviously, the direction of this effective field is influenced by the DLT, otherwise the $x$ component of the final state should be zero. Because the effective-field components stemming from the SOT are mainly determined by SOT current density, we can expect that the DLT has the same effect on the total effective field if $J_{\mathrm{SOT}} \geq J_{\text {th }}$. Figure 2(b) shows the trajectory when $J_{\mathrm{SOT}}=69 \mathrm{MA} \mathrm{cm}^{-2}$ is applied. We can see that the precession amplitude gradually increases and $\mathbf{m}$ finally stops in the $x-y$ plane, but the effective-field direction, denoted by the red star in Fig. 2(d), is nearly the same since the applied SOT current density is close. Note that we only stress the effect of the DLT on the effective field at the beginning of switching. When $\mathbf{m}$ finally stops at the $x-y$ plane, or to be more specific, along the $-y$ direction, $H_{\mathrm{SOT}}^{\mathrm{DL}}(\mathbf{m} \times \boldsymbol{\sigma} \times \mathbf{m})$ becomes zero, thus $H_{x}$ is still required to break the symmetry of SOT.

It is not straightforward to determine the direction of $\mathbf{H}_{\text {eff }}$ from Eq. (3) since $\mathbf{m}$ enters the expression of it. Instead, we adopt the final state of magnetization as the direction of $\mathbf{H}_{\text {eff, }}$ which is the static solution of Eq. (3). Note that the final state here refers to the case when $J_{\text {SOT }}<J_{\text {th }}$. But as discussed before, the effective field of the case when $J_{\text {SOT }} \geq J_{\text {th }}$ can be estimated by the circumstance when $J_{\text {SOT }}<J_{\text {th }}$ as long as their current densities are almost the same. In the case of $(\partial \mathbf{m} / \partial t)=0$, Eq. (3) reduces to

$$
\begin{aligned}
& -\gamma \mu_{0}\left(\mathbf{m} \times \mathbf{H}_{\mathbf{K}, \mathbf{e f f}}\right)-\gamma \mu_{0}\left(\mathbf{m} \times \mathbf{H}_{\mathbf{x}}\right) \\
& \quad+\gamma \mu_{0} H_{\mathrm{SOT}}^{\mathrm{DL}}(\mathbf{m} \times \boldsymbol{\sigma} \times \mathbf{m})+\gamma \mu_{0} H_{\mathrm{SOT}}^{\mathrm{FL}}(\mathbf{m} \times \boldsymbol{\sigma})=0,
\end{aligned}
$$


(a)

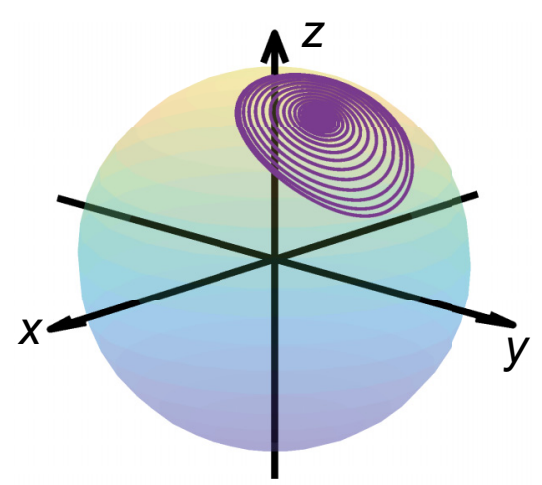

(c)

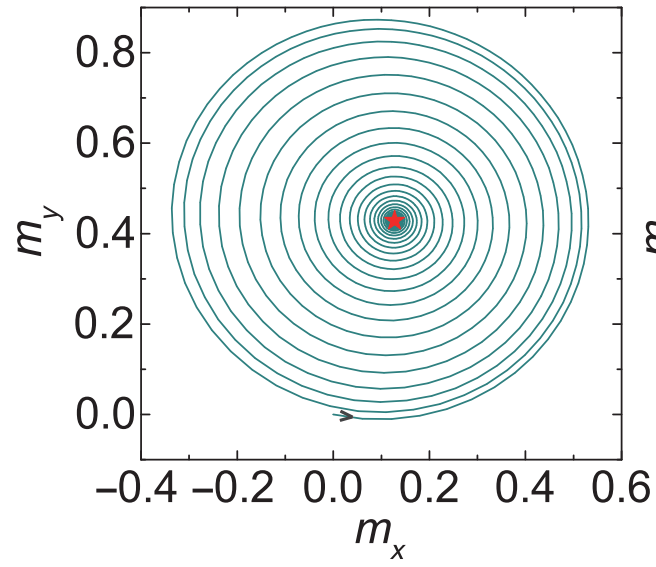

which is further simplified to the following form:

$$
\mathbf{m} \times \mathbf{H}_{\text {eff }}=0,
$$

(d)
FIG. 2. Tridimensional trajectory of magnetization $\boldsymbol{m}$ when (a) $J_{\mathrm{SOT}}=68 \mathrm{MA} \mathrm{cm}^{-2}$ and (b) $J_{\text {SOT }}=69 \mathrm{MA} \mathrm{cm}^{-2}$. Trajectory of $\boldsymbol{m}$ projecting on the $x-y$ plane when (c) $J_{\mathrm{SOT}}=68 \mathrm{MA} \mathrm{cm}^{-2}$ and (d) $J_{\mathrm{SOT}}=69 \mathrm{MA} \mathrm{cm}^{-2} \cdot \alpha=0.1$ and $\eta=3$. The red star in (c) and (d) denotes the final state of $\boldsymbol{m}$, i.e., the effective-field direction, when $J_{\mathrm{SOT}}=68 \mathrm{MA} \mathrm{cm}^{-2}$.

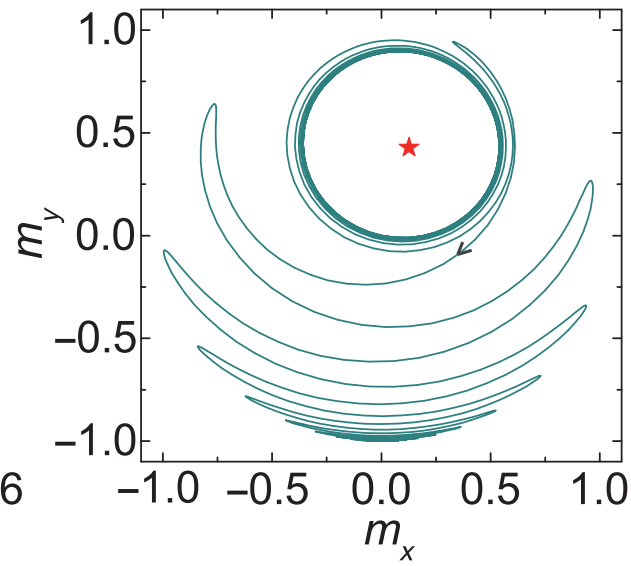

where $\quad \mathbf{H}_{\mathrm{eff}}=\mathbf{H}_{\mathbf{K} \text {,eff }}+\mathbf{H}_{\mathbf{x}}-H_{\mathrm{SOT}}^{\mathrm{DL}}(\boldsymbol{\sigma} \times \mathbf{m})-H_{\mathrm{SOT}}^{\mathrm{FL}} \boldsymbol{\sigma}$. Adopting the spherical coordinates, the direction of $\mathbf{H}_{\text {eff }}$ is approximately given by (see Appendix A for details):

$$
\left\{\begin{array}{l}
\sin \theta_{H}=\sqrt{\frac{d}{1-\frac{1}{2} d-c^{2}+b^{2}}} \\
\sin \varphi_{H}=\frac{\eta c}{\sqrt{d}}
\end{array},\right.
$$

where $b=H_{x} / H_{K \text {,eff, }} c=H_{\mathrm{SOT}}^{\mathrm{DL}} / H_{K, \text { eff }}, d=\left(1+\eta^{2}\right) c^{2}+2 b c+b^{2} . \theta_{H}$ and $\varphi_{H}$ are the polar angle and azimuthal angle of $\mathbf{H}_{\text {eff }}$, respectively. Then the analytical formula of threshold SOT current density $J_{\text {th }}$ is derived (see Appendix B for details) as

$$
J_{\mathrm{th}}=\frac{e t_{F} \mu_{0} M_{s} H_{K, \mathrm{eff}}}{\hbar \theta_{\mathrm{SH}}} \frac{\sqrt{4 \alpha\left(4 \alpha+2 \alpha \eta^{2}+\eta\right)+\left(9 \alpha^{2}-4 \alpha \eta-8 \alpha^{2} \eta^{2}\right) b^{2}}-5 \alpha b}{4 \alpha+2 \alpha \eta^{2}+\eta} .
$$

When $H_{x}<<H_{K, \text { eff }}(b<<1)$, Eq. (7) is further simplified to

$$
J_{\mathrm{th}}=\frac{e t_{F} \mu_{0} M_{s} H_{K, \mathrm{eff}}}{\hbar \theta_{\mathrm{SH}}}\left(\sqrt{\frac{4 \alpha}{4 \alpha+2 \alpha \eta^{2}+\eta}}-\frac{5 \alpha b}{4 \alpha+2 \alpha \eta^{2}+\eta}\right) \text {. }
$$

In contrast, when $\eta<0$, there is no direct competition between SOT and damping [32], indicating that we should obtain the analytical expression from Eq. (4) [26]. We first neglect the in-plane field $H_{x}$ in Eq. (4) 
and obtain

$$
\sin \theta \sqrt{\eta^{2}+\cos ^{2} \theta}-\left(1+\eta^{2}\right) c=0,
$$

where $c=H_{\mathrm{SOT}}^{\mathrm{DL}} / H_{K, \text { eff }}$ and $\theta$ is the polar angle of magnetization $\mathbf{m}$. Though $\theta$ is mathematically equivalent to the $\theta_{H}$ above, we use a different symbol to show the difference between the two derivations. The threshold current density is deduced from the condition that no $\theta \in(0, \pi / 2)$ satisfies Eq. (9). Therefore, we obtain

$$
J_{\text {th }}=\left\{\begin{array}{c}
\frac{e t_{F} \mu_{0} M_{S} H_{K, \text { eff }}}{\hbar \theta_{\mathrm{SH}}},-1<\eta<0 \\
-\frac{2 \eta}{1+\eta^{2}} \frac{e t_{F} \mu_{0} M_{S} H_{K, \mathrm{eff}}}{\hbar \theta_{\mathrm{SH}}}, \eta \leq-1
\end{array} .\right.
$$

The formula containing $H_{x}$ has not been directly derived. Instead, since numerical simulations [see Fig. 4(b)] demonstrate good linearity between the $J_{\text {th }}$ and $H_{x}$, we include the effect of $H_{x}$ by modifying Eq. (10) as

$$
J_{\mathrm{th}}{ }^{*}=J_{\mathrm{th}}-k b \frac{e t_{F} \mu_{0} M_{S} H_{K, \mathrm{eff}}}{\hbar \theta_{\mathrm{SH}}},
$$

where $b=H_{x} / H_{K \text {,eff }}$ and the coefficient $k$ is a function of $\eta$ fitted by

$$
k=0.66 \frac{6.83-\eta}{(\eta+0.81)^{2}+2.92} .
$$

\section{RESULTS AND DISCUSSION}

\section{A. Validation of formulas $\boldsymbol{J}_{\text {th }}$ with numerical results}

We now compare the derived $J_{\text {th }}$ with numerical results. Figures 3(a) and 3(b) show the comparison of Eqs. (1), (2), and (7) with macrospin simulation results when $\eta>0$. We can see that Eq. (7) shows good coincidence with the numerical values, especially when $\alpha$ is small and $\eta$ is large. In contrast, distinct deviations can be observed. This is because the simplifications during the derivation are stable in the former condition, where the magnetization is reversed in a precessional manner. However, in the latter case, $\boldsymbol{m}$ will damp rapidly into the final state. In particular, the switching process completes within one precession when $\eta=0$. Nevertheless, Eq. (7) shows higher accuracy than Eqs. (1) and (2), which indicates that, for one thing, the effect of FLT on SOT threshold current density $J_{\text {th }}$ cannot be neglected, even if FLT is not strong; for another, the contribution of DLT to the effective field is not negligible, especially when FLT is weak. We further verify the reliability of Eqs. (7) and (8) among a wide range of $\alpha$ and $\eta$ when $\mu_{0} H_{x}=40 \mathrm{mT}$. Figure 3(c) presents the errors of Eq. (7), which is defined as $\left[\left(J_{\text {th }}-J_{\text {num }}\right) / J_{\text {num }}\right] \times 100 \%$ with $J_{\text {num }}$ denoting the numerical results when $\alpha$ varies from 0.004 to 0.128 and $\eta$ ranges from 0.2 to 3 . The errors are less than $13 \%$ among the whole regime and even within $6.6 \%$ when $\eta \geq 0.4$, showing the high accuracy of our formula. The relative difference between Eqs. (7) and (8),
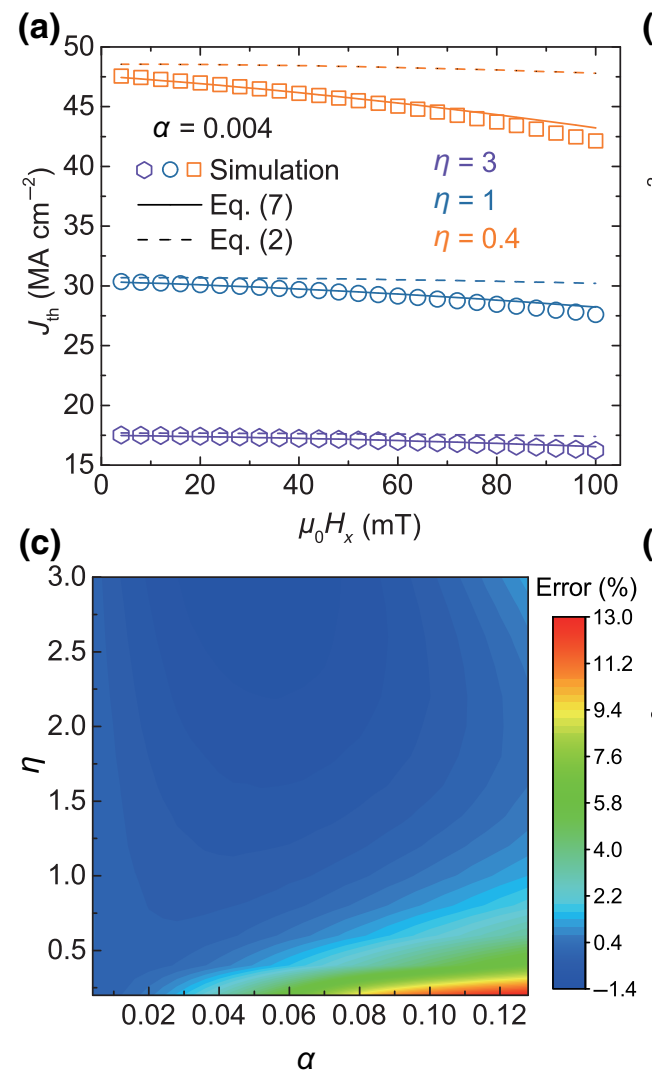

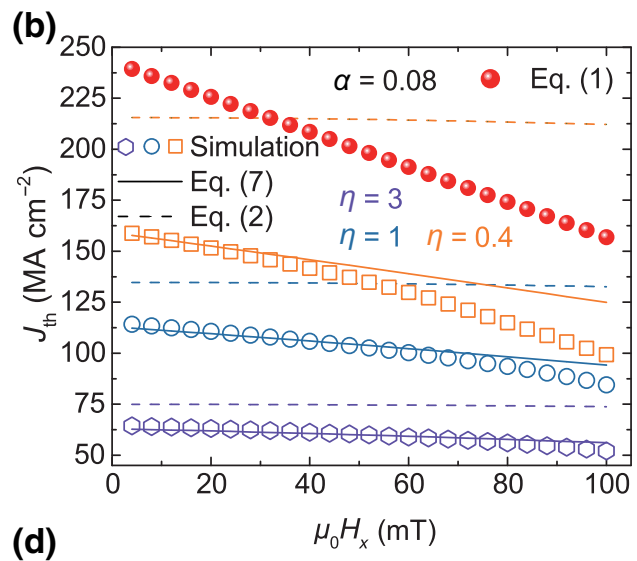

(d)

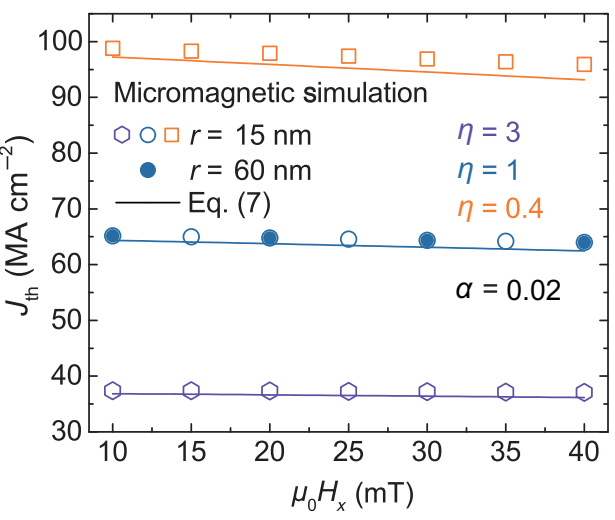

FIG. 3. Comparison of Eqs. (1), (2), and (7) with macrospin simulations when (a) $\alpha=0.004$, (b) $\alpha=0.08$ with various $\eta$ and $H_{x}$. The colors correspond to different $\eta$. (c) Errors of Eq. (7) compared to numerical results among a wide range of $\alpha$ and $\eta . \mu_{0} H_{x}=40 \mathrm{mT}$ is used. (d) Comparison of Eq. (7) with micromagnetic simulations when $\alpha=0.02$. The MTJ radius $r$ is set as 15 and $60 \mathrm{~nm}$, respectively. 
defined as $\{[$ Eq. (8) - Eq. (7)]/Eq. (7) $\} \times 100 \%$ is calculated to be less than $0.5 \%$ (not shown), thus the simplified Eq. (8) is applicable in the low field regime. The accuracy of Eq. (7) is also verified by micromagnetic simulations, as shown in Fig. 3(d). To investigate the switching beginning from domain-wall nucleation, we set the radius of MTJs $r$ as $60 \mathrm{~nm}$. Interestingly, the calculated threshold current density in devices with $r=60 \mathrm{~nm}$ still agrees well with Eq. (7), indicating that the current density required to nucleate a domain wall can be reduced when a FLT with $\eta>0$ is incorporated [41].

The formulas derived under $\eta<0$ are in good agreement with numerical results as well. As displayed in Fig. 4(a), Eq. (10) basically depicts the trend of $J_{\text {th }}$ with increasing $|\eta|$, but deviations occur when the in-plane field is applied. Therefore, we numerically calculate the $J_{\text {th }}$ under different $H_{x}$ and $\eta$ [See Fig. 4(b)]. The good linearity between $J_{\text {th }}$ and $H_{x}$ allows us to consider the effect of $H_{x}$ using Eq. (11). The coefficient $k$ with different $\eta$ is well fitted by Eq. (12), as shown in Fig. 4(c). Subsequently, we can see that the macrospin simulation results in Figs. 4(a) and 4(b) show good coincidence with Eq. (11). Again, the reliability of Eq. (11) is validated via micromagnetic simulations, as indicated in Fig. 4(d). Different from the $\eta>0$ case, the switching is coherent even in the devices with $r=60 \mathrm{~nm}$. In contrast, a moderate DMI of $0.2 \mathrm{~mJ} \mathrm{~m}^{-2}$ together with the FLT favor a domain wall nucleating at the edge firstly and then propagating [17]. Still, we can see that Eq. (11) appears as a good approximation of the threshold current density.

\section{B. Conditions to achieve complete switching}

Though the switching dynamics are totally different when the sign of $\eta$ changes, the derived formulas indicate that a large FLT, for example, $|\eta|=3$, can be utilized to effectively reduce the required SOT current density. However, as discussed previously, these formulas are the threshold to switch magnetization to the nearly in-plane states while complete switching cannot be ensured after SOT currents are turned off. To investigate the condition to realize stable switching, we calculate the phase diagram of magnetization after a trapezoidal SOT pulse with a width of $300 \mathrm{~ns}$ following a relaxation time of $50 \mathrm{~ns}$ through macrospin simulations. $\mu_{0} H_{x}$ ranges from 3 to $150 \mathrm{mT}$. As shown in Fig. 5(a), whether the switching is achieved or not depends delicately on the applied SOT current density $J_{\text {SOT }}$ and the in-plane magnetic field $H_{x}$ when $\eta=2, \alpha=0.02$. But different from the case with $\eta=0$, there is no region to realize stable switching with $J_{\text {SOT }}$ and $H_{x}$ higher than their threshold values [33]. Instead, because the switching-back phenomenon originates from the applied in-plane field, we can use a small field $H_{x}$, whose threshold is indicated by the red arrow in Fig. 5(a), to ensure the stable switching. Alternatively, Fig. 5(b) shows that we can expand the stable switching regime by (a)

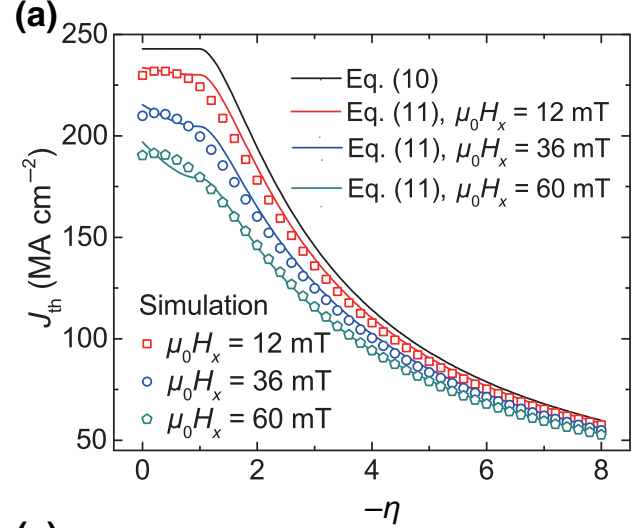

(c)

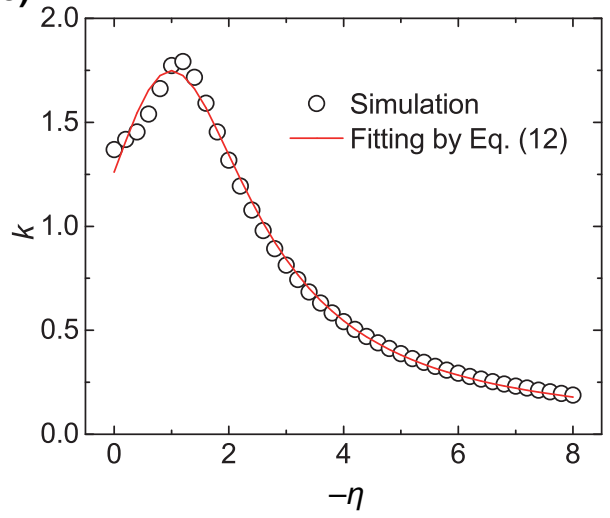

(b)

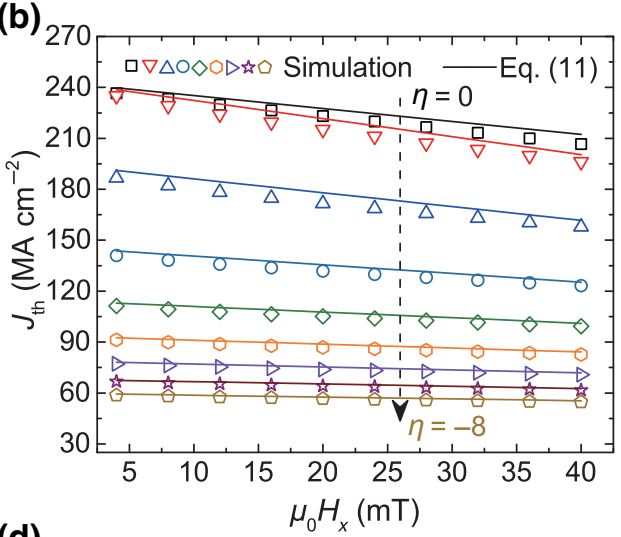

(d)

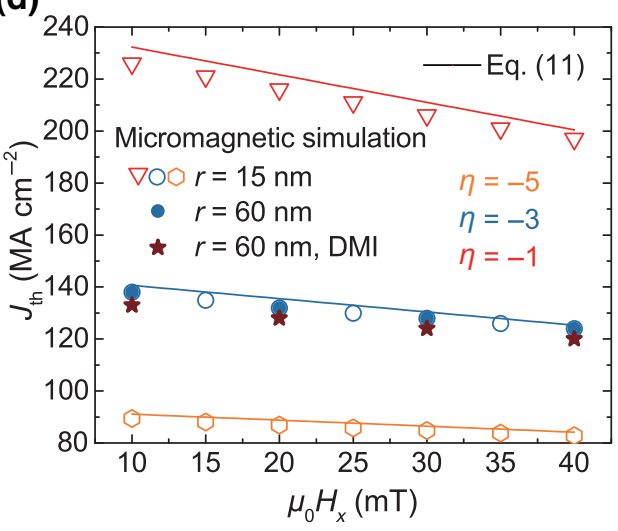

FIG. 4. (a) Comparison of Eqs. (10) and (11) with macrospin simulations when $\eta$ varies from 0 to -8 and $\mu_{0} H_{x}=12,36$, and $60 \mathrm{mT}$, respectively. (b) Comparison of Eq. (11) with macrospin simulations when $\mu_{0} H_{x}$ ranges from 4 to $40 \mathrm{mT}$ and $\eta$ varies from 0 to -8 . (c) Fitting of the coefficient $k$ by Eq. (12). (d) Comparison of Eq. (11) with micromagnetic simulations under different $\eta$. The MTJ radius $r$ is set as 15 and $60 \mathrm{~nm}$, respectively. The DMI strength is $0.2 \mathrm{~mJ} \mathrm{~m}^{-2}$. The damping constant $\alpha$ is set as 0.1 in the above simulations because there is no competition between SOT and damping when $\eta<0$. To satisfy the quasistatic Eq. (4), the applied SOT pulses possess a rise time $T_{\text {rise }}$ of $0.5 \mathrm{~ns}$. 
virtue of a high damping [26]. The maximum in-plane field $\mu_{0} H_{x}^{\max }$ under different damping constant is summarized in Fig. 5(c). The $H_{x}^{\max }$ can be approximately given by $H_{x}^{\max }=$ $\alpha H_{K \text {,eff }}$ when $\eta=0$. The FLT only has negligible influence on the $H_{x}^{\max }$ if the fall time of SOT pulses $T_{\text {fall }}=0 \mathrm{~ns}$, but obviously decrease the $H_{x}^{\max }$ when $T_{\text {fall }}=0.2 \mathrm{~ns}$, which is explained afterwards.

The phase diagram with $\eta=-2$ is in sharp contrast with the $\eta=2$ case. As shown in Fig. 5(d), stable switching is attained among all the $H_{x}$ when $J_{\text {SOT }}$ is slightly higher than the threshold. However, switching back occurs with increasing SOT current density, as also demonstrated by the yellow curve in Fig. 5(f). We can adopt the same scheme as the $\eta=2$ case, but we find that a fall time of $0.2 \mathrm{~ns}$ can be used to promote complete switching [see Fig. 5(e)]. The reason is explained as follows. The changing rate of the $z$ component of magnetization $m_{z}$ depends on the competition of various torques. We assume that $\mathbf{m} \sim\left(\delta m_{x},-1, \delta m_{z}\right)$ when SOT currents begin to decrease, which is typically valid when $J_{\mathrm{SOT}}$ is strong enough. The sign of $\delta m_{x}$ depends on various parameters but $\delta m_{z}<0$ is doubtless because of the effect of $H_{x}$. At this moment, SOT can be neglected because $\mathbf{m}$ lies nearly in the direction of $\boldsymbol{\sigma}$. Meanwhile, $-\gamma \mu_{0}\left(\mathbf{m} \times \mathbf{H}_{\mathbf{K} \text {,eff }}\right)$ and $-\gamma \mu_{0}\left(\mathbf{m} \times \mathbf{H}_{\mathbf{x}}\right)$ rotate the magnetization anticlockwise around $-z$ and $+x$ direction, respectively. As a consequence, both $m_{x}$ and $m_{z}$ are decreasing ( $m_{x}$ becomes negative), which is consistent with curves in Fig. 5(f). If the fall time is long enough, i.e., $J_{\text {SOT }}$ is still strong when $m_{x}$ becomes negative and non-negligible, the FLT promotes or suppresses stable switching, depending on the sign of $\eta$. To be more specific, when $\eta<0$, the $z$ component of the FLT $\left.\gamma \mu_{0} \eta H_{\mathrm{SOT}}^{\mathrm{DL}}(\mathbf{m} \times \boldsymbol{\sigma})\right|_{z}$ is negative, promoting magnetization switching to $-z$ direction. In contrast, FLT with $\eta>0$ favors switching back when $T_{\text {fall }} \neq 0$, thus $H_{x}^{\text {max }}$ should be smaller in this case, as shown in Fig. 5(c). In our simulations, $T_{\text {fall }}=0.2 \mathrm{~ns}$ is long enough to ensure stable switch-

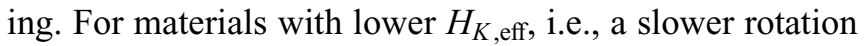
rate around $-z$ direction at the beginning, longer $T_{\text {fall }}$ is needed.
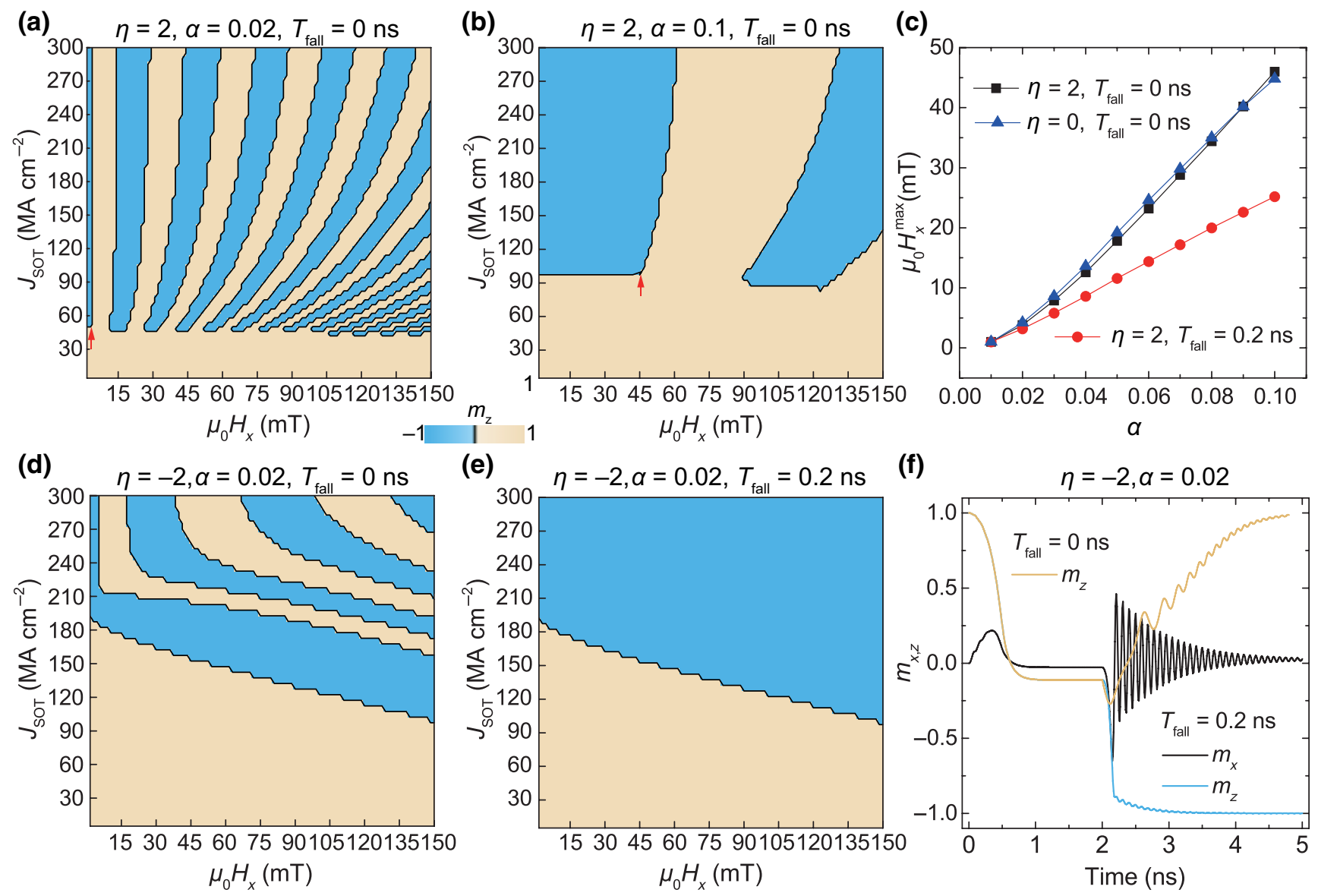

FIG. 5. Phase diagram of $m_{z}$ after applying a trapezoidal SOT pulse of $300 \mathrm{~ns}$ following by a relaxation time of $50 \mathrm{~ns}$ when $\eta=2, T_{\text {fall }}=0$ ns and (a) $\alpha=0.02$, (b) $\alpha=0.1$. (c) The maximum in-plane field $\mu_{0} H_{x}^{\max }$ under different $\alpha$ to ensure stable switching when $\eta=2$. Phase diagram of magnetization after the same excitation and relaxation when $\eta=-2, \alpha=0.02$ and (d) $T_{\text {fall }}=0$ ns, (e) $T_{\text {fall }}=0.2 \mathrm{~ns}$. (f) Switching dynamics with different fall time when $\eta=-2, \alpha=0.02, \mu_{0} H_{x}=10 \mathrm{mT}$ and $J_{\mathrm{SOT}}=215 \mathrm{MA} \mathrm{cm}^{-2}$. The rise time, steady time of the SOT pulse are 0.5 and $1.5 \mathrm{~ns}$, respectively, thus the SOT current density begins to decrease at $t=2 \mathrm{~ns}$. 
TABLE I. Experiments interpreted by Eqs. (1), (2), (7), and (8).

\begin{tabular}{lllcccc}
\hline \hline & \multicolumn{5}{c}{$J_{\text {th }}\left(\mathrm{MA} \mathrm{cm}^{-2}\right)$} \\
\cline { 2 - 7 } Materials & Diameter & Measured & Eq. (1) & Eq. (2) & Eq. (7) & Eq. (8) \\
\hline $\mathrm{W} /(\mathrm{Co}, \mathrm{Fe}) \mathrm{B}[18]$ & $62 \mathrm{~nm}$ & $78.9^{\mathrm{a}}$ & 109.3 & 124.8 & 80.8 & 80.8 \\
$\mathrm{Ta} /(\mathrm{Co}, \mathrm{Fe}) \mathrm{B}[19]$ & $30 \mathrm{~nm}$ & 400 & 1346.3 & 583.4 & 481.4 & 482.7 \\
\hline \hline
\end{tabular}

${ }^{a}$ We estimate this value from Fig. 6 in Ref. [18] as it was not explicitly given.

Based on the conditions discussed above, we then apply the derived formulas to interpret the measured threshold current densities in two experiments, where the magnetization switching is roughly single domain $[18,19]$, i.e., the lateral size of magnetic free layers is less than approximately $60 \mathrm{~nm}$. It should be noted that the microscopic origin of SOT and the sign of $\eta$ are still under debate. While the theory for spin Hall effect supports a negative sign of $\eta$ [42], recent experiments have demonstrated that $\eta$ is positive in $\mathrm{Ta}(6 \mathrm{~nm}) /(\mathrm{Co}, \mathrm{Fe}) \mathrm{B}(0.8 \mathrm{~nm}) / \mathrm{MgO}$ $(2 \mathrm{~nm})$ [43], consistent with the Rashba mechanism [44]. In addition, Takeuchi et al. found that there is no evident dependence between the FLT in $\mathrm{W} /(\mathrm{Co}, \mathrm{Fe}) \mathrm{B} / \mathrm{MgO}$ and the thickness of $\mathrm{W}$ [45], indicating the necessity of interfacial mechanism. Therefore, we use Eqs. (7) and (8), where $\eta$ is positive, to analyze the experiments in $\mathrm{Ta}(\mathrm{W}) /(\mathrm{Co}, \mathrm{Fe}) \mathrm{B} / \mathrm{MgO}$ systems $[18,19]$. Besides, according to the in-plane magnetic field used in their experiments, damping constant $\alpha=0.08$ is used to ensure stable switching while other parameters are adopted from experiments (see Appendix $\mathrm{C}$ for other parameters). As shown in Table I, these experimental results can be well interpreted by Eqs. (7) and (8), especially in $\mathrm{Ta} /(\mathrm{Co}, \mathrm{Fe}) \mathrm{B} / \mathrm{MgO}$ where the FLT is strong. We can also see that the accuracy of Eq. (2) cannot be ensured if the FLT is weak and a relatively high damping is used. We should note that the typical damping constant measured in $\mathrm{Ta}(\mathrm{W}) /(\mathrm{Co}, \mathrm{Fe}) \mathrm{B} / \mathrm{MgO}$ is about $0.02-0.03[7,46]$. Using $\alpha=0.03$, the experimental results can be approximately reproduced by Eqs. (2), (7), and (8), but it remains uncovered how stable complete switching is realized in this case. If the damping constant can be enhanced during the injection of SOT currents, further experiments are required to elucidate the underlying mechanism.

\section{Incubation delay of SOT-induced magnetization switching}

Magnetization switching induced by SOT has been considered faster than STT because the spin polarization of SOT $\sigma$ is orthogonal to the initial magnetization $\mathbf{m}_{\mathbf{0}}$, thus SOT reaches its maximum on the outset. As a consequence, it is generally accepted that there is no incubation delay in SOT switching [21], which has been experimentally evidenced in $\mathrm{Pt} / \mathrm{Co} / \mathrm{AlO} x[16,17]$.
In the meantime, subnanosecond switching has been demonstrated in $\mathrm{Ta}(\mathrm{W}) /(\mathrm{Co}, \mathrm{Fe}) \mathrm{B} / \mathrm{MgO}$ systems $[18,20]$. However, as indicated in Ref. [32], SOT with $\eta>0$ competes directly with damping, which induces multiple precessions at the beginning. In contrast, SOT with $\eta \leq 0$ does not compete with damping, resulting in nonprecessional behavior. These magnetization dynamics are reproduced in our macrospin simulations, as shown in Figs. 2(b) and 5(f). Therefore, it is possible that incubation time can emerge during SOT-induced magnetization switching when $\eta>0$. Interestingly, incubation delay up to several nanoseconds is observed in the $\mathrm{W} /(\mathrm{Co}, \mathrm{Fe}) \mathrm{B} / \mathrm{MgO}$ system [34], where $\eta$ could be positive as discussed above.

We further verify the incubation delay via micromagnetic simulations. Figure 6 shows the evolution of $m_{z}$ in devices with $r=60 \mathrm{~nm}$ when $\eta=0.5, \alpha=0.05$, $J_{\text {SOT }}=135 \mathrm{MA} \mathrm{cm}^{-2}$ and $\mu_{0} H_{x}=15 \mathrm{mT}$. Multiple precessions of $m_{z}$ can be observed before $t=\sim 19 \mathrm{~ns}$, consistent with the macrospin simulations. In addition, the magnetization is nearly coherent until $t=\sim 20 \mathrm{~ns}$ when domain-wall nucleation occurs. The magnetization stays in an intermediate state before the SOT current is switched off at $t=25 \mathrm{~ns}$ [47]. Afterwards, the magnetization relaxes to the $-z$ direction under the joint effect of damping and $H_{x}$. But it is worth noting that the incubation delay, which imposes a limitation for STT switching speed, will not become a major issue to SOT switching thanks to the capacity of heavy metal to apply high currents. Applying $J_{\text {SOT }}=200 \mathrm{MA} \mathrm{cm}^{-2}$, magnetization switching can be realized through a 0.7 -ns SOT pulse, which is consistent with the experiments in Ref. [34]. The incubation delay can also be eliminated in materials with a strong damping constant because the precessions brought by FLT at the beginning are canceled by damping (see Appendix D). Therefore, it makes sense that no observable incubation delay is found in $\mathrm{Pt} / \mathrm{Co} / \mathrm{AlO} x$, where the damping constant $\alpha$ is as large as $0.1-0.3$ [48]. Another interpretation is that $\eta$ is negative in such a material system. Combined with DMI and the FLT, the switching dynamics in their experiments, such as no incubation delay and domain-wall nucleation at four quadrants, can be well reproduced [17].

To date, it is still a challenge to achieve energy-efficient SOT switching [34]. From the derived Eqs. (7) and (11), we see the potential to reduce SOT current density to about $\sqrt{4 \alpha /\left(4 \alpha+2 \alpha \eta^{2}+\eta\right)}$ and $2|\eta| /\left(1+\eta^{2}\right)$ with FLT 


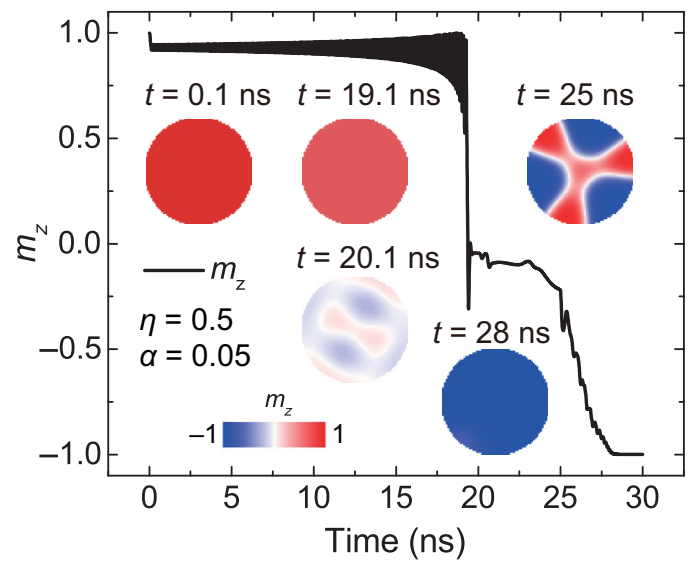

FIG. 6. Evolution of $m_{z}$ in devices with $r=60 \mathrm{~nm}$. Simulations parameters are $\eta=0.5, \alpha=0.05, J_{\text {SOT }}=135 \mathrm{MA}$ $\mathrm{cm}^{-2}$ and $\mu_{0} H_{x}=15 \mathrm{mT}$. Insets are magnetization snapshots at selected times. $T_{\text {rise }}=0.1 \mathrm{~ns}$ is used in the simulation.

compared to switching by solely DLT. Intuitively, the $\eta>0$ case exhibits a higher reduction rate, especially when the damping constant is low. However, to realize subnanosecond switching, which is the expectation of SOT, a relatively high current is still required to eliminate the incubation delay. Considering also the switching-back issue, material systems with large FLT $(\eta>0)$ and moderate damping, for example, $\eta=3$ and $\alpha=0.05$, are more suitable to realize simultaneously the low power and stable switching. Micromagnetic simulations show that complete magnetization switching is feasible using a 0.7-ns SOT pulse with a density of $100 \mathrm{MA} \mathrm{cm}^{-2}$ and $\mu_{0} H_{x}$ of $15 \mathrm{mT}$ in this case $\left(J_{\text {th }}\right.$ is approximately $230 \mathrm{MA} \mathrm{cm}{ }^{-2}$ when no FLT is included). Switching schemes with no in-plane magnetic fields, such as interface-generated spin currents in ferromagnetic trilayers [29] and the interplay of STT and SOT [27], can be used to resolve the switching-back issue. In contrast, SOT with $\eta<0$ results in fast switching even when $J_{\mathrm{SOT}}$ is just slightly higher than the threshold because of the vanishing of incubation delay. The stability is enhanced by the fall time of SOT pulses as well. Complete magnetization switching using a 0.5 -ns pulse is realized using $\eta=-8$ and $J_{\mathrm{SOT}}=70 \mathrm{MA} \mathrm{cm}{ }^{-2}$ by micromagnetic simulations. With the precondition to engineer materials with giant $|\eta|$, the $\eta<0$ case appears more promising.

\section{CONCLUSIONS}

We derive analytical expressions of the SOT threshold current density to switch perpendicular magnetization from equilibrium states to nearly in-plane states. These formulas agree well with numerical results among a wide range of simulated parameters and indicate the potential to reduce SOT current density with a large FLT. Conditions for stable magnetization switching after removing
SOT currents are obtained. We find that a long enough fall time of SOT pulses ensures stable switching for the $\eta<0$ case while in contrast a small in-plane magnetic field or a high damping is required when $\eta>0$. We further apply the derived formulas to interpret several experiments well. In addition, we investigate the possible incubation delay induced by SOT, clarifying the contradiction in recent experiments. Our findings help deepen the understanding of existing experiments and provide guidance for both theoretical and experimental SOT research.

\section{ACKNOWLEDGMENTS}

The authors are thankful for the support from the National Natural Science Foundation of China (Grants No. 61571023, No. 61627813, and No. 51602013), the International Collaboration Project B16001, and the National Key Technology Program of China 2017ZX01032101.

\section{APPENDIX A: DETERMINATION OF THE EFFECTIVE-FIELD DIRECTION}

Based on the discussion in Sec. II, the effective-field direction $\left(\theta_{H}, \varphi_{H}\right)$ is determined by

$$
\mathbf{m} \times \mathbf{H}_{\text {eff }}=0 .
$$

Since $\mathbf{H}_{\text {eff }}$ can be expressed as $\left(H_{\mathrm{SOT}}^{\mathrm{DL}} m_{z}+H_{x}, \eta H_{\mathrm{SOT}}^{\mathrm{DL}}\right.$, $\left.H_{K, \text { eff }} m_{z}-H_{\mathrm{SOT}}^{\mathrm{DL}} m_{x}\right)$ with $m_{i}(i=x, y, z)$ denoting the $i$ component of $\mathbf{m}$, Eq. (A1) is equivalent to

$$
\left\{\begin{array}{l}
H_{\mathrm{SOT}}^{\mathrm{DL}} m_{z}+H_{x}=g m_{x} \\
\eta H_{\mathrm{SOT}}^{\mathrm{DL}}=g m_{y} \\
H_{K, \mathrm{eff}} m_{z}-H_{\mathrm{SOT}}^{\mathrm{DL}} m_{x}=g m_{z}
\end{array},\right.
$$

where $g$ is a proportional constant.

We first consider the case with $H_{x}=0$ because it is easier to analyze. Combining the first and third equation of Eq. (A2), we obtain the following quadratic equation of $g$ :

$$
g^{2}-g H_{K, \text { eff }}+\left(H_{\mathrm{SOT}}^{\mathrm{DL}}\right)^{2}=0 .
$$

The solution of Eq. (A3) exists when $H_{K, \text { eff }}^{2}-4\left(H_{\text {SOT }}^{\text {DL }}\right)^{2} \geq$ 0 which translates to

$$
H_{\mathrm{SOT}}^{\mathrm{DL}} \leq 0.5 H_{K, \text { eff }} \quad \text { or } \quad J_{\mathrm{SOT}} \leq \frac{e t_{F} \mu_{0} M_{s} H_{K, \text { eff }}}{\hbar \theta_{\mathrm{SH}}}=J_{C 0} .
$$

We can see that $J_{C 0}$ is the upper bound of the SOT threshold current density to destabilize magnetization from equilibrium states, as also indicated by Eqs. (1), (7), (10), and (11). In the absence of FLT, $H_{\mathrm{SOT}}^{\mathrm{DL}}$ is close to $0.5 H_{K \text {,eff, }}$ resulting in a largely tilted $\mathbf{H}_{\text {eff }}$.

When $H_{x}$ is included, it is difficult to give an accurate solution to Eq. (A2). Assume $m_{z} \sim 1$, 


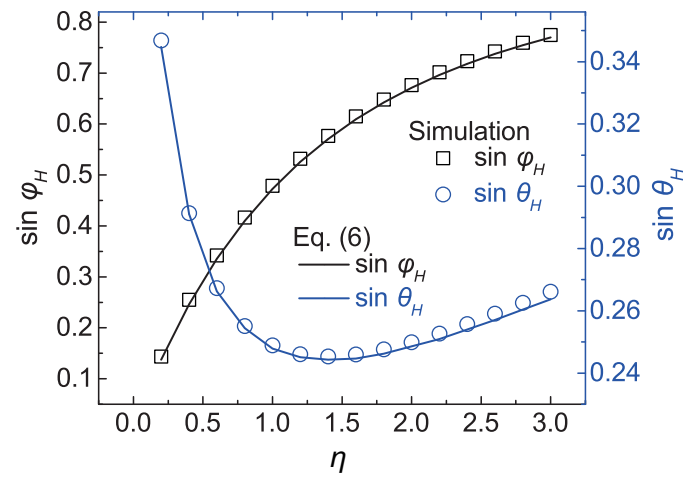

FIG. 7. Verification of Eq. (6) with $J_{\text {SOT }}=0.99 J_{\text {th }}$ for each data point when $\alpha=0.016$ and $\mu_{0} H_{x}=20 \mathrm{mT}$. The boxes and circles show the numerical results while the curves show the calculated values using Eq. (6).

which is accurate enough when $\eta$ is strong and $\alpha$ is small, we get $m_{x}=\left(H_{\mathrm{SOT}}^{\mathrm{DL}}+H_{x}\right) / g$ from the first equation of Eq. (A2). Then from the second the third equations of Eq. (A2), we get $m_{y}=\eta H_{\mathrm{SOT}}^{\mathrm{DL}} / g$ and $m_{z}=$ $\left[H_{\mathrm{SOT}}^{\mathrm{DL}}\left(H_{\mathrm{SOT}}^{\mathrm{DL}}+H_{x}\right)\right] /\left[\left(H_{K, \text { eff }}-g\right) g\right]$. Using $m_{x}^{2}+m_{y}^{2}+$ $m_{z}^{2}=1$ and the assumption $g \sim H_{K \text {,eff }}$ indicated in Eq. (A3) when $\eta$ is strong and $\alpha$ is small, we obtain

$$
g^{*}=1-\frac{c(c+b)}{\sqrt{1-d}}
$$

where $g^{*}=g / H_{K \text {,eff }}, b=H_{x} / H_{K, \text { eff }}, c=H_{\mathrm{SOT}}^{\mathrm{DL}} / H_{K, \text { eff }}, d=$ $\left(1+\eta^{2}\right) c^{2}+2 b c+b^{2}$. Then, combining Eqs. (A2), (A5) and $m_{x}^{2}+m_{y}^{2}+m_{z}^{2}=1$, we get

$$
m_{z}=\sqrt{\frac{\sqrt{1-d}-2 c(c+b)-\eta^{2} t^{2} \sqrt{1-d}}{\sqrt{1-d}-2 c(c+b)+(c+b)^{2} / \sqrt{1-d}}},
$$

which can be further simplified using the assumption $d<<1$ :

$$
m_{z}=\sqrt{\frac{1-\frac{3}{2} d-c^{2}+b^{2}}{1-\frac{1}{2} d-c^{2}+b^{2}}} .
$$

Considering the first and second equation of Eq. (A2), we obtain

$$
\tan \varphi_{H}=\frac{\eta H_{\mathrm{SOT}}^{\mathrm{DL}}}{H_{\mathrm{SOT}}^{\mathrm{DL}} m_{z}+H_{x}}=\frac{\eta c}{c m_{z}+b} .
$$

By virtue of Eq. (A7) and ignoring the terms $b^{m} c^{n}$ with $m+n \geq 3$, we get Eq. (6) in the paper, which agrees well with numerical results, as shown in Fig. 7.

\section{APPENDIX B: DERIVATION OF THE ANALYTICAL EXPRESSIONS OF $\boldsymbol{J}_{\text {th }}$}

To derive the analytic expressions, Eq. (3) in Sec. II is firstly transformed to

$$
\begin{aligned}
\frac{\partial \mathbf{m}}{\partial t}= & \frac{-\gamma \mu_{0}}{1+\alpha^{2}}\left[\left(\mathbf{m} \times \mathbf{H}_{\mathbf{K}, \mathbf{e f f}}\right)+\alpha \mathbf{m} \times\left(\mathbf{m} \times \mathbf{H}_{\mathbf{K}, \mathbf{e f f}}\right)\right. \\
& +\left(\mathbf{m} \times \mathbf{H}_{\mathbf{x}}\right)+\alpha \mathbf{m} \times\left(\mathbf{m} \times \mathbf{H}_{\mathbf{x}}\right) \\
& -(1-\alpha \eta) H_{\mathrm{SOT}}^{\mathrm{DL}}(\mathbf{m} \times \boldsymbol{\sigma} \times \mathbf{m}) \\
& \left.-(\alpha+\eta) H_{\mathrm{SOT}}^{\mathrm{DL}}(\mathbf{m} \times \boldsymbol{\sigma})\right] .
\end{aligned}
$$

With $\left(\theta_{H}, \varphi_{H}\right)$ determined, we adopt a new coordinate $x^{\prime} y^{\prime} z^{\prime}$ with $z^{\prime}$ along the direction of $\mathbf{H}_{\text {eff. }}$ The rotation matrix from coordinate $x y z$ to $x^{\prime} y^{\prime} z^{\prime}$ is given by

$$
R=\left(\begin{array}{ccc}
\cos \theta_{H} & 0 & -\sin \theta_{H} \\
0 & 1 & 0 \\
\sin \theta_{H} & 0 & \cos \theta_{H}
\end{array}\right)\left(\begin{array}{ccc}
\cos \varphi_{H} & \sin \varphi_{H} & 0 \\
-\sin \varphi_{H} & \cos \varphi_{H} & 0 \\
0 & 0 & 1
\end{array}\right)
$$

Given that the initial magnetization $\boldsymbol{m}_{\mathbf{0}}=(0,0,1)$ in coordinate $x y z$, we can obtain $\mathbf{m}_{0}{ }^{\prime}=\left(-\sin \theta_{H}, 0, \cos \theta_{H}\right)$ in coordinate $x^{\prime} y^{\prime} z^{\prime}$. Assuming that the $z^{\prime}$ component of magnetization $\mathbf{m}^{\prime}$ remains unchanged at the beginning and $\sin ^{2} \theta_{H} \sim 0$, we can linearize Eq. (B1) by the following simplifications:

$$
\left\{\begin{array}{l}
m_{p}^{\prime} m_{q}^{\prime}=0, \quad p, q=x, y \\
m_{z}^{\prime}=\cos \theta_{H}
\end{array} .\right.
$$

Consequently, Eq. (B1) is transformed to a second-order ordinary differential equation

$$
\frac{1+\alpha^{2}}{\gamma \mu_{0}} \frac{d}{d t}\left(\begin{array}{l}
m_{x^{\prime}} \\
m_{y^{\prime}}
\end{array}\right)=\mathbf{M}\left(\begin{array}{l}
m_{x^{\prime}} \\
m_{y^{\prime}}
\end{array}\right)+\mathbf{G},
$$

where $\mathbf{M}$ is a $2 \times 2$ matrix and $\mathbf{G}$ is a column vector as

$$
\left\{\begin{array}{l}
\left.M_{11}=\alpha H_{K, \mathrm{eff}} \sin ^{2} \theta_{H}-\cos ^{4} \theta_{H}\right)+(1-\alpha \eta) H_{\mathrm{SOT}}^{\mathrm{DL}} \sin \theta_{H} \cos \theta_{H} \sin \varphi_{H}-\alpha H_{x} \sin \theta_{H} \cos \theta_{H} \cos \varphi_{H} \\
M_{12}=-H_{K, \mathrm{eff}} \cos ^{3} \theta_{H}-(\alpha+\eta) H_{\mathrm{SOT}}^{\mathrm{DL}} \sin \theta_{H} \sin \varphi_{H}-H_{x} \sin \theta_{H} \cos \varphi_{H} \\
M_{21}=H_{K, \mathrm{eff}} \cos ^{3} \theta_{H}-H_{K, \mathrm{eff}} \cos ^{2} \theta_{H} \sin \theta_{H}+(\alpha+\eta) H_{\mathrm{SOT}}^{\mathrm{DL}} \sin \theta_{H} \sin \varphi_{H}+H_{x} \sin \theta_{H} \cos \varphi_{H} \\
M_{22}=-\alpha H_{K, \mathrm{eff}} \cos ^{4} \theta_{H}+(1-\alpha \eta) H_{\mathrm{SOT}}^{\mathrm{DL}} \sin \theta_{H} \cos \theta_{H} \sin \varphi_{H}-\alpha H_{x} \sin \theta_{H} \cos \theta_{H} \cos \varphi_{H}
\end{array}\right.
$$


TABLE II. Detailed parameters adopted in the calculation of Table I.

\begin{tabular}{lllllll}
\hline \hline & \multicolumn{5}{c}{ Parameters } \\
\cline { 2 - 6 } Materials & $t_{F}$ & $\mu_{0} H_{K, \text { eff }}$ & $M_{s}\left(\mathrm{~A} \mathrm{~m}^{-1}\right)$ & $\left|\theta_{\mathrm{SH}}\right|$ & $\eta$ & $\mu_{0} H_{x}$ \\
\hline $\mathrm{W} /(\mathrm{Co}, \mathrm{Fe}) \mathrm{B}[18]$ & $1 \mathrm{~nm}$ & $0.29 \mathrm{~T}$ & $9 \times 10^{5}$ & 0.32 & 0.31 & $24 \mathrm{mT}$ \\
$\mathrm{Ta} /(\mathrm{Co}, \mathrm{Fe}) \mathrm{B}[19]$ & $1.2 \mathrm{~nm}$ & $0.25 \mathrm{~T}$ & $1 \times 10^{6}$ & 0.03 & 2 & $20 \mathrm{mT}$ \\
\hline \hline
\end{tabular}

$$
\left\{\begin{array}{l}
G_{1}=-\alpha H_{K, \text { eff }} \sin \theta_{H} \cos ^{2} \theta_{H}-(1-\alpha \eta) H_{\mathrm{SOT}}^{\mathrm{DL}} \cos \theta_{H} \sin \varphi_{H}-(\alpha+\eta) H_{\mathrm{SOT}}^{\mathrm{DL}} \cos \theta_{H} \cos \varphi_{H} \\
\quad-H_{x} \cos \theta_{H} \sin \varphi_{H}+\alpha H_{x} \cos \theta_{H} \cos \varphi_{H} \\
G_{2}=H_{K, \text { eff }} \sin \theta_{H} \cos ^{3} \theta_{H}+(1-\alpha \eta) H_{\mathrm{SOT}}^{\mathrm{DL}} \cos \varphi_{H}-(\alpha+\eta) H_{\mathrm{SOT}}^{\mathrm{DL}} \cos ^{2} \theta_{H} \sin \varphi_{H} \\
\quad+H_{x} \cos ^{2} \theta_{H} \cos \varphi_{H}-\alpha H_{x} \sin \varphi_{H}
\end{array} .\right.
$$

The characteristic root of $\mathbf{M}$ is calculated as $\lambda_{1,2}=$ $\left[\left(M_{11}+M_{22}\right) \pm i(\sqrt{-\Delta})\right] / 2$, with $\Delta=\left(M_{11}-M_{22}\right)^{2}+$ $4 M_{12} M_{21}<0$. Therefore, whether the solution to Eq. (B4) diverges or converges, i.e., whether switching can be realized or not, depends on the sign of $M_{11}+M_{22}$ and the boundary condition is $M_{11}+M_{22}=0$, which is equivalent to

$$
\begin{gathered}
2(1-\alpha \eta) H_{\mathrm{SOT}}^{\mathrm{DL}} \sin \theta_{H} \cos \theta_{H} \sin \varphi_{H} \\
\quad+2 \alpha H_{x} \sin \theta_{H} \cos \theta_{H} \cos \varphi_{H} \\
=\alpha H_{K, \mathrm{eff}}\left(2 \cos ^{4} \theta_{H}-\sin ^{2} \theta_{H}\right) .
\end{gathered}
$$

By substituting the expression of $\theta_{H}$ and $\varphi_{H}$ into Eq. (B7), the analytic expression of threshold current density, i.e., Eq. (7) in Sec. III, is obtained.

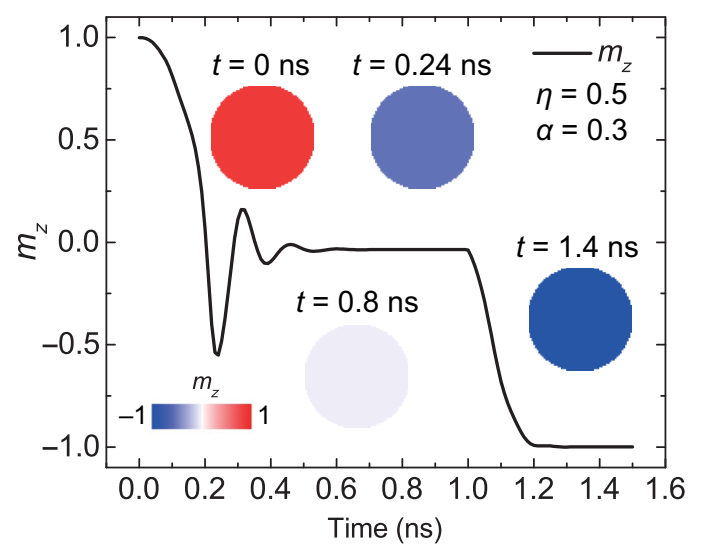

FIG. 8. Evolution of $m_{z}$ in devices with $r=60 \mathrm{~nm}$. Simulations parameters are $\eta=0.5, \alpha=0.3, J_{\mathrm{SOT}}=215 \mathrm{MA} \mathrm{cm}^{-2}$ and $\mu_{0} H_{x}=15 \mathrm{mT}$. Insets are magnetization snapshots at selected times. $T_{\text {rise }}=0.1 \mathrm{~ns}$ is used in the simulation. Note that $J_{\text {SOT }}$ used here is just slightly higher than the threshold current density.

\section{APPENDIX C: PARAMETERS USED IN THE CALCULATION OF TABLE I}

The detailed parameters utilized in the calculation of Table I in the manuscript are summarized in Table II. We do not find the value of $M_{s}$ in Ref. [19]. But using $M_{s}=1 \times 10^{6} \mathrm{~A} \mathrm{~m}^{-1}$, the calculated $J_{\text {th }}$ through Eq. (2) is close to $1400 \mathrm{MA} \mathrm{cm}^{-2}$ reported in Ref. [19].

\section{APPENDIX D: INCUBATION ELIMINATED BY HIGH DAMPING}

Figure 8 shows that the incubation delay induced by SOT with $\eta>0$ can be eliminated in materials with high damping. Interestingly, unlike the case with $\alpha=0.05$, magnetization switching is nearly coherent, indicating that high damping can suppress domain-wall nucleation within devices. However, when DMI $=0.4 \mathrm{~mJ} \mathrm{~m}^{-2}$ is included, the switching starts from domain-wall nucleation at the edge firstly, which is attributed to the joint effect of DMI and FLT [17].

[1] M. M. Waldrop, The chips are down for Moore's law, Nature 530, 144 (2016).

[2] A. D. Kent and D. C. Worledge, A new spin on magnetic memories, Nat. Nanotechnol. 10, 187 (2015).

[3] S. Bhatti, R. Sbiaa, A. Hirohata, H. Ohno, S. Fukami, and S. N. Piramanayagam, Spintronics based random access memory: A review, Mater. Today 20, 530 (2017).

[4] R. Ramaswamy, J. Lee, K. Cai, and H. Yang, Recent advances in spin-orbit torques: Moving towards device applications, Appl. Phys. Rev. 5, 031107 (2018).

[5] S. Peng, D. Zhu, J. Zhou, B. Zhang, A. Cao, M. Wang, W. Cai, K. Cao, and W. Zhao, Modulation of heavy metal/ferromagnetic metal interface for high-performance spintronic devices, Adv. Electron. Mater 5, 1900134 (2019).

[6] J. C. Slonczewski, Current-driven excitation of magnetic multilayers, J. Magn. Magn. Mater 159, L1 (1996). 
[7] S. Ikeda, K. Miura, H. Yamamoto, K. Mizunuma, H. D. Gan, M. Endo, S. Kanai, J. Hayakawa, F. Matsukura, and H. Ohno, A perpendicular-anisotropy $\mathrm{CoFeB}-\mathrm{MgO}$ magnetic tunnel junction, Nat. Mater. 9, 721 (2010).

[8] M. Wang, W. Cai, K. Cao, J. Zhou, J. Wrona, S. Peng, H. Yang, J. Wei, W. Kang, Y. Zhang, J. Langer, B. Ocker, A. Fert, and W. Zhao, Current-induced magnetization switching in atom thick tungsten engineered perpendicular magnetic tunnel junctions with large tunnel magnetoresistance, Nat. Commun. 9, 671 (2018).

[9] G. Hu, J. J. Nowak, M. G. Gottwald, J. Z. Sun, D. Houssameddine, J. Bak, S. L. Brown, P. Hashemi, Q. He, J. Kim, C. Kothandaraman, G. Lauer, H. K. Lee, T. Suwannasiri, P. L. Trouilloud, and D. C. Worledge, Reliable five-nanosecond writing of spin-transfer torque magnetic random-access memory, IEEE Magn. Lett. 10, 4504304 (2019).

[10] X. Wang, Z. Wang, X. Hao, Y. Zhou, J. Zhang, H. Gan, D. Jung, K. Satoh, B. Yen, R. Malmhall, and Y. Huai, Different dielectric breakdown mechanisms for RF $\mathrm{MgO}$ and naturally oxidized MgO, Appl. Phys. Expr. 7, 083002 (2014).

[11] T. Devolder, C. Chappert, J. A. Katine, M. J. Carey, and K. Ito, Distribution of the magnetization reversal duration in subnanosecond spin-transfer switching, Phys. Rev. B 75, 064402 (2007).

[12] L. Liu, O. J. Lee, T. J. Gudmundsen, D. C. Ralph, and R. A. Buhrman, Current-Induced Switching of Perpendicularly Magnetized Magnetic Layers Using Spin Torque from the Spin Hall Effect, Phys. Rev. Lett. 109, 096602 (2012).

[13] L. Liu, C. Pai, Y. Li, H. W. Tseng, D. C. Ralph, and R. A. Buhrman, Spin-torque switching with the giant spin Hall effect of tantalum, Science 336, 555 (2012).

[14] I. M. Miron, G. Gaudin, S. Auffret, B. Rodmacq, A. Schuhl, S. Pizzini, J. Vogel, and P. Gambardella, Current-driven spin torque induced by the Rashba effect in a ferromagnetic metal layer, Nat. Mater. 9, 230 (2010).

[15] Y. Fan, P. Upadhyaya, X. Kou, M. Lang, S. Takei, Z. Wang, J. Tang, L. He, L. Chang, M. Montazeri, G. Yu, W. Jiang, T. Nie, R. N. Schwartz, Y. Tserkovnyak, and K. L. Wang, Magnetization switching through giant spin-orbit torque in a magnetically doped topological insulator heterostructure, Nat. Mater. 13, 699 (2014).

[16] K. Garello, C. O. Avci, I. M. Miron, M. Baumgartner, A. Ghosh, S. Auffret, O. Boulle, G. Gaudin, and P. Gambardella, Ultrafast magnetization switching by spin-orbit torques, Appl. Phys. Lett. 105, 212402 (2014).

[17] M. Baumgartner, K. Garello, J. Mendil, C. O. Avci, E. Grimaldi, C. Murer, J. Feng, M. Gabureac, C. Stamm, Y. Acremann, S. Finizio, S. Wintz, J. Raabe, and P. Gambardella, Spatially and time-resolved magnetization dynamics driven by spin-orbit torques, Nat. Nanotechnol. 12, 980 (2017).

[18] K. Garello, F. Yasin, S. Couet, L. Souriau, J. Swerts, S. Rao, S. Van Beek, W. Kim, E. Liu, S. Kundu, D. Tsvetanova, K. Croes, N. Jossart, E. Grimaldi, M. Baumgartner, D. Crotti, A. Fumemont, P. Gambardella, and G. S. Kar, in 2018 IEEE Symposium on VLSI Circuits (Honolulu, HI, 2018), pp. 81.
[19] C. Zhang, S. Fukami, H. Sato, F. Matsukura, and H. Ohno, Spin-orbit torque induced magnetization switching in nanoscale Ta/CoFeB/MgO, Appl. Phys. Lett. 107, 012401 (2015).

[20] M. Cubukcu, O. Boulle, N. Mikuszeit, C. Hamelin, T. Brächer, N. Lamard, M. Cyrille, L. Buda-Prejbeanu, K. Garello, I. M. Miron, O. Klein, G. Loubens, V. V. Naletov, J. Langer, B. Ocker, P. Gambardella, and G. Gaudin, Ultrafast perpendicular spin-orbit torque MRAM, IEEE Trans. Magn. 54, 9300204 (2018).

[21] A. Manchon, J. Železný, I. M. Miron, T. Jungwirth, J. Sinova, A. Thiaville, K. Garello, and P. Gambardella, Current-induced spin-orbit torques in ferromagnetic and antiferromagnetic systems, Rev. Mod. Phys. 91, 035004 (2019).

[22] P. M. Haney, H.-W. Lee, K.-J. Lee, A. Manchon, and M. D. Stiles, Current induced torques and interfacial spin-orbit coupling: Semiclassical modeling, Phys. Rev. B 87, 174411 (2013).

[23] P. M. Haney, H.-W. Lee, K.-J. Lee, A. Manchon, and M. D. Stiles, Current-induced torques and interfacial spin-orbit coupling, Phys. Rev. B 88, 214417 (2013).

[24] X. Fan, H. Celik, J. Wu, C. Ni, K.-J. Lee, V. O. Lorenz, and J. Q. Xiao, Quantifying interface and bulk contributions to spin-orbit torque in magnetic bilayers, Nat. Commun. 5, 3042 (2014).

[25] A. Ghosh, K. Garello, C. O. Avci, M. Gabureac, and P. Gambardella, Interface-Enhanced Spin-Orbit Torques and Current-Induced Magnetization Switching of $\mathrm{Pd} / \mathrm{Co} / \mathrm{AlOx}$, Phys. Rev. Appl. 7, 014004 (2017).

[26] K.-S. Lee, S.-W. Lee, B.-C. Min, and K.-J. Lee, Threshold current for switching of a perpendicular magnetic layer induced by spin Hall effect, Appl. Phys. Lett. 102, 112410 (2013).

[27] M. Wang, W. Cai, D. Zhu, Z. Wang, J. Kan, Z. Zhao, K. Cao, Z. Wang, Y. Zhang, T. Zhang, C. Park, J.-P. Wang, A. Fert, and W. Zhao, Field-free switching of a perpendicular magnetic tunnel junction through the interplay of spin-orbit and spin-transfer torques, Nat. Electron. 1, 582 (2018).

[28] G. Yu, P. Upadhyaya, Y. Fan, J. G. Alzate, W. Jiang, K. L. Wong, S. Takei, S. A. Bender, L. Chang, Y. Jiang, M. Lang, J. Tang, Y. Wang, Y. Tserkovnyak, P. K. Amiri, and K. L. Wang, Switching of perpendicular magnetization by spin-orbit torques in the absence of external magnetic fields, Nat. Nanotechnol. 9, 548 (2014).

[29] S. C. Baek, V. P. Amin, Y.-W. Oh, G. Go, S.-J. Lee, G.H. Lee, K.-J. Kim, M. D. Stiles, B.-G. Park, and K.-J. Lee, Spin currents and spin-orbit torques in ferromagnetic trilayers, Nat. Mater. 17, 509 (2018).

[30] K. Cai, M. Yang, H. Ju, S. Wang, Y. Ji, B. Li, K. Edmonds, Y. Sheng, B. Zhang, N. Zhang, S. Liu, H. Zheng, and K. Wang, Electric field control of deterministic currentinduced magnetization switching in a hybrid ferromagnetic/ferroelectric structure, Nat. Mater. 16, 712 (2017).

[31] S. Łazarski, W. Skowroński, J. Kanak, Ł Karwacki, S. Ziętek, K. Grochot, T. Stobiecki, and F. Stobiecki, FieldFree Spin-Orbit-Torque Switching in $\mathrm{Co} / \mathrm{Pt} / \mathrm{Co}$ Multilayer with Mixed Magnetic Anisotropies, Phys. Rev. Appl. 12, 014006 (2019). 
[32] T. Taniguchi, S. Mitani, and M. Hayashi, Critical current destabilizing perpendicular magnetization by the spin Hall effect, Phys. Rev. B 92, 024428 (2015).

[33] T. Taniguchi, Theoretical condition for switching the magnetization in a perpendicularly magnetized ferromagnet via the spin Hall effect, Phys. Rev. B 100, 174419 (2019).

[34] E. Grimaldi, V. Krizakova, G. Sala, F. Yasin, S. Couet, G. Kar, K. Garello, and P. Gambardella, Single-shot dynamics of spin-orbit torque and spin transfer torque switching in three-terminal magnetic tunnel junctions, Nat. Nanotechno. 15, 111 (2020).

[35] J. Park, G. E. Rowlands, O. J. Lee, D. C. Ralph, and R. A. Buhrman, Macrospin modeling of sub-ns pulse switching of perpendicularly magnetized free layer via spin-orbit torques for cryogenic memory applications, Appl. Phys. Lett. 105, 102404 (2014).

[36] J. Kim, J. Sinha, M. Hayashi, M. Yamanouchi, S. Fukami, T. Suzuki, S. Mitani, and H. Ohno, Layer thickness dependence of the current-induced effective field vector in TaCoFeBMgO, Nat. Mater. 12, 240 (2013).

[37] N. Sato, A. El-Ghazaly, R. M. White, and S. Wang, Effect of $\mathrm{Mg}$ oxidation degree on Rashba-effect-induced torques in $\mathrm{Ta} / \mathrm{CoFeB} / \mathrm{Mg}(\mathrm{MgO})$ multilayer, IEEE Trans. Magn. 52, 1400304 (2016).

[38] C. O. Avci, K. Garello, C. Nistor, S. Godey, B. Ballesteros, A. Mugarza, A. Barla, M. Valvidares, E. Pellegrin, A. Ghosh, I. M. Miron, O. Boulle, S. Auffret, G. Gaudin, and P. Gambardella, Fieldlike and antidamping spin-orbit torques in as-grown and annealed $\mathrm{Ta} / \mathrm{CoFeB} / \mathrm{MgO}$ layers, Phys. Rev. B 89, 214419 (2014).

[39] A. Vansteenkiste, J. Leliaert, M. Dvornik, M. Helsen, F. Sanchez, and B. Waeyenberge, The design and verification of MuMax3, AIP Adv. 4, 107133 (2014).
[40] K.-S. Lee, S.-W. Lee, B.-C. Min, and K.-J. Lee, Thermally activated switching of perpendicular magnet by spin-orbit spin torque, Appl. Phys. Lett. 104, 072413 (2014).

[41] W. Fan, J. Zhao, M. Tang, H. Chen, H. Yang, W. Lü, Z. Shi, and X. Qiu, Asymmetric Spin-Orbit-Torque-Induced Magnetization Switching with a Noncollinear in-Plane Assisting magnetic Field, Phys. Rev. Appl. 11, 034018 (2019).

[42] K. Xia, P. J. Kelly, G. E. W. Bauer, A. Brataas, and I. Turek, Spin torques in ferromagnetic/normal-metal structures, Phys. Rev. B 65, 220401 (2002).

[43] J. Yoon, S.-W. Lee, J. H. Kwon, J. M. Lee, J. Son, X. Qiu, K.-J. Lee, and H. Yang, Anomalous spin-orbit torque switching due to field-like torque-assisted domain wall reflection, Sci. Adv. 3, e1603099 (2017).

[44] H. Kurebayashi, J. Sinova, D. Fang, A. C. Irvine, T. D. Skinner, J. Wunderlich, V. Novák, R. P. Campion, B. L. Gallagher, E. K. Vehstedt, L. P. Zârbo, K. Výborný, A. J. Ferguson, and T. Jungwirth, An antidamping spin-orbit torque originating from the Berry curvature, Nat. Nanotechnol. 9, 211 (2014).

[45] Y. Takeuchi, C. Zhang, A. Okada, H. Sato, S. Fukami, and Hideo Ohno, Spin-orbit torques in high-resistivityW/CoFeB/MgO, Appl. Phys. Lett. 112, 192408 (2018).

[46] D. M. Lattery, D. Zhang, J. Zhu, X. Hang, J.-P. Wang, and $\mathrm{X}$. Wang, Low Gilbert damping constant in perpendicularly magnetized $\mathrm{W} / \mathrm{CoFeB} / \mathrm{MgO}$ films with high thermal stability, Sci. Rep. 8, 13395 (2018).

[47] G. Finocchio, M. Carpentieri, E. Martinez, and B. Azzerboni, Switching of a single ferromagnetic layer driven by spin Hall effect, Appl. Phys. Lett. 102, 212410 (2013).

[48] A. J. Schellekens, L. Deen, D. Wang, J. T. Kohlhepp, H. J. M. Swagten, and B. Koopmans, Determining the Gilbert damping in perpendicularly magnetized $\mathrm{Pt} / \mathrm{Co} / \mathrm{AlOx}$ films, Appl. Phys. Lett. 102, 082405 (2013). 